\title{
Inkjet Printed Patch Antennas on Transparent Substrates
}

\author{
Tursunjan Yasin* and Reyhan Baktur \\ Department of Electrical and Computer Engineering, \\ Utah State University, Logan UT 84322, USA \\ E-mail: reyhan.baktur@usu.edu
}

\section{Introduction}

There is a growing interest in optically transparent antennas. Turpin et al. has demonstrated a meshed patch antenna with $93 \%$ transparency where the antenna could be either fabricated from electroformed metal mesh or screen printed on transparent substrates with conductive inks [1]. For a faster and more accurate fabrication, it is favorable to print antenna with an inkjet printer as described by Rida [2]. While Rida's approach focused mainly on using paper substrates and on ring or dipole type antennas, this paper is aimed to present a cheaper printing method and the feasibility of printing patch type antennas on transparent substrates for the applications such as solar cell integration.

\section{Rectangular Solid Patch Antenna}

As an initial feasibility study, a solid rectangular patch antenna (Fig. 1-a) was printed on a Polyethylene Terephthalate (PET) transparency using an Epson C88+ printer and silver conductive ink (JS-15) from Novacentrix. After curing, the antenna is assembled on a Rogers substrate (RO4003, thickness $=1.524 \mathrm{~mm}$ ) without the top conductor layer. Two pieces of copper tapes were used to tune the antenna and to provide a better connection with the SMA connector. For comparison, another antenna (labeled as copper) is fabricated on the same substrate with a LPKH circuit board milling machine. The antennas were designed to operate at $2.5 \mathrm{GHz}$ with ground planes of $72 \mathrm{~mm}$ by $80 \mathrm{~mm}$. It is seen that the printed antenna has a shift in the frequency (Fig. 1-b) but this is mainly due to the additional layer of PET. Fig. 2 shows the measured radiation patterns and it is clear that the performance of two antennas is comparable. There is only a difference of $0.725 \mathrm{~dB}$ in gain between the two antennas.

\section{Circular Meshed Patch Antenna}

The study in the previous section suggested the feasibility of fabricating antennas with more complicated geometry using inkjet printing technique. The following study is to demonstrate printing of circular meshed patch antennas. The feed geometry is as shown in Fig. 3-a where the meshed antenna is excited from its side using coupling lines. This type of feeding is simple and effective in matching the antenna to a $50 \mathrm{Ohm}$ microstrip line, and one can simultaneously print the feed together with the antenna design. Ansoft's HFSS was used to perform initial 
simulation without considering the loss of the conductive ink. The simulated results were then used as guidelines for fabrication. The initial values for the coupling distance, length and the width of the brunch lines (Fig. 3-a) were taken referencing Zhu [3] and Liu [4], and then were refined during the simulation.

To validate the feed design and antenna performance, two meshed circular patch antennas were printed using the same printing essential as in Section 2. The PET substrate with the antenna printed on it was assembled a Plexiglas (thickness $=2.032 \mathrm{~mm}, \varepsilon_{\mathrm{r}}=2.6$, loss tangent $=0.0057$ ) that serves as the substrate for the meshed antenna. The Plexiglas is then backed by copper tape that serves as the ground plane (Fig. 3-b). The size of the ground plane is $150 \mathrm{~mm}$ by $170 \mathrm{~mm}$. This assembly is chosen because in application where the meshed antenna is integrated on solar panels, the metal layer on the solar panel is solid and does not have to be transparent. Both antennas were designed to operate at around $2.5 \mathrm{GHz}$ and have optical transparency of $60 \%$. The difference between two antenna geometry is the width of mesh lines. One antenna has more refined lines of 0.72 $\mathrm{mm}$ and the other one has mesh lines of $1.35 \mathrm{~mm}$.

\section{Conclusions}

The paper presents the feasibility of printing patch type antennas with a commercial inkjet printer. The printing method is especially valuable for complex and fine antenna geometry. As examples, solid rectangular and meshed circular patch antennas were printed and measured results show good antenna performance. Although the study experimented with the antenna of transparency of $60 \%$, the fabrication and feed design presented can be easily applied to antennas with transparency higher than $90 \%$, allowing one to integrate these antennas with solar cells, windows, and LED displays.

\section{Acknowledgement}

We thank Novacentrix for providing technical support and material supply. We also thank Rogers Corporation for supplying high frequency laminate.

\section{References}

[1] Timothy W. Turpin, and R. Baktur, "Integrated Solar Cell Meshed Patch Antennas", to appear on IEEE Antennas and Wireless Propagation Letters, 8: 693-696, 2009.

[2] Amin H. Rida, Conductive Inkjet Printed Antennas on Flexible Low-cost Paper-based Substrates for RFID and WSN Applications, Master Thesis, Georgia Institute of Technology, May 2009. 
[3] Lei ZHU and $\mathrm{Ke}$ Wu, "Line-to-ring Coupling Circular Model and Its Parametric Effects for Optimized Design of Microstrip Ring Circuits and Antennas", 1997 IEEE MTT-S Digest Volume: 1, page(s): 289-292.

[4] Ji-Chyun Liu, Hui Chi Wu, Meng Shan Chiang, Hsin-Yi Tsou, "T-coupled Circular Microstrip Antenna with H-shaped Aperture for Bandwidth and Radiation-gain Improvements", Microwave and Optical Technology Letters, Volume 37 Issue 6, Pages $414-417$

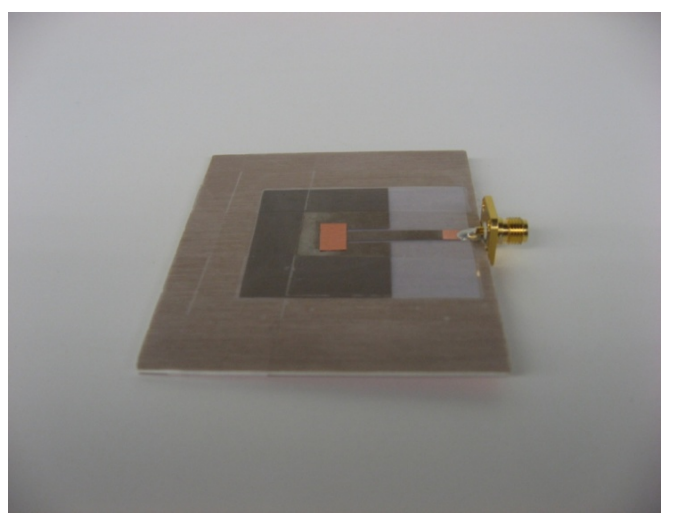

(a)

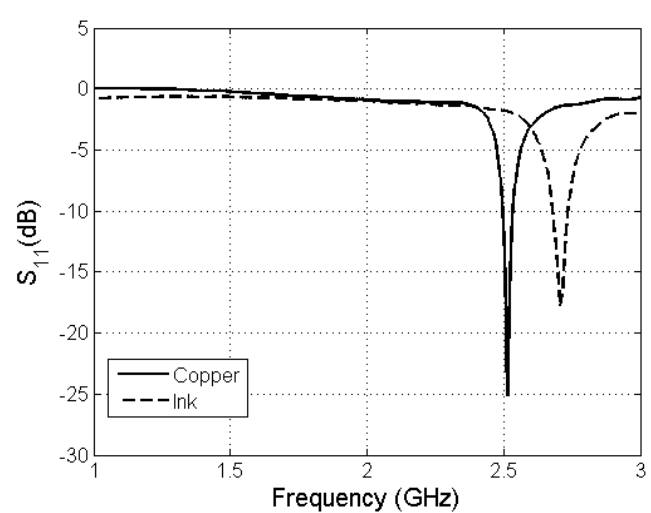

(b)

Fig. 1: (a) An inkjet printed rectangular patch antenna, (b) Measured return loss of the inkjet printed antenna and an antenna fabricated from circuit board milling machine.

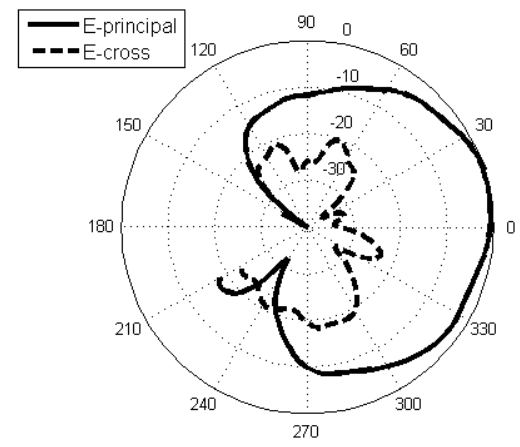

(a)

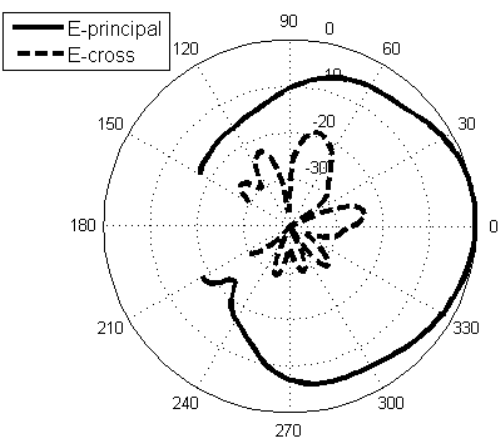

(b)

Fig. 2: Normalized E-plane pattern (co- and cross-polarization) of printed antenna (a) and the anenna fabricated with circuit board milling machine (b). 


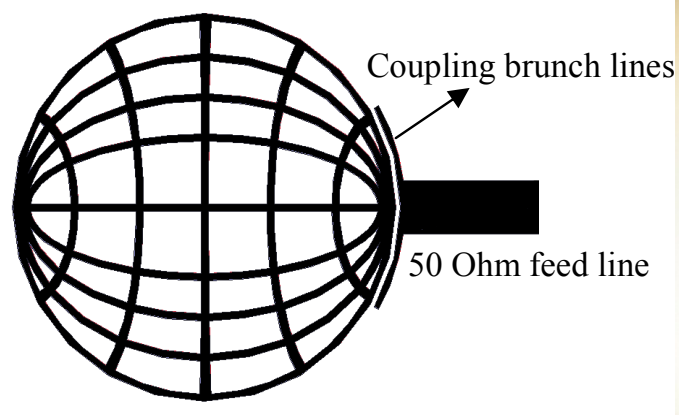

(a) Geometry of a meshed circular patch antenna with side-coupled line feed

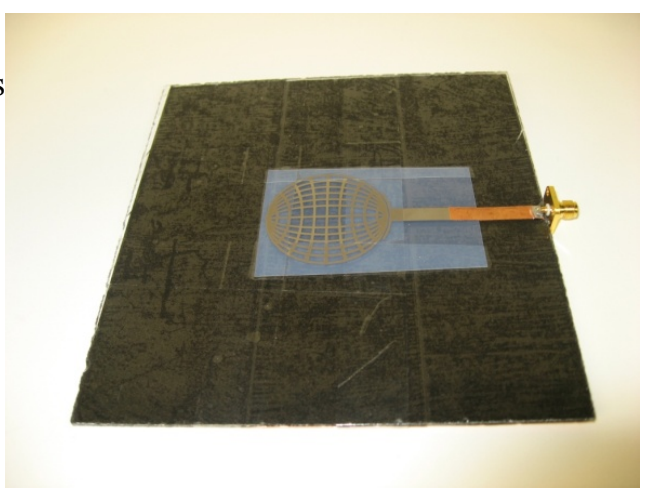

(b) Inkjet printed circular meshed patch antenna on plexiglass substrate backed with the copper tape conductor

Fig. 3: Circular meshed patch antenna

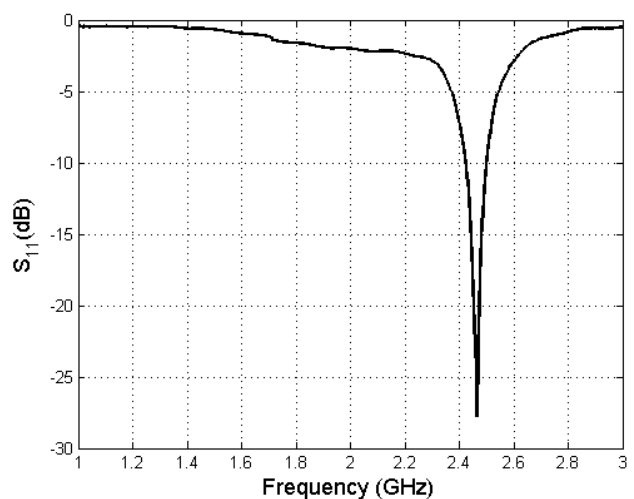

(a)

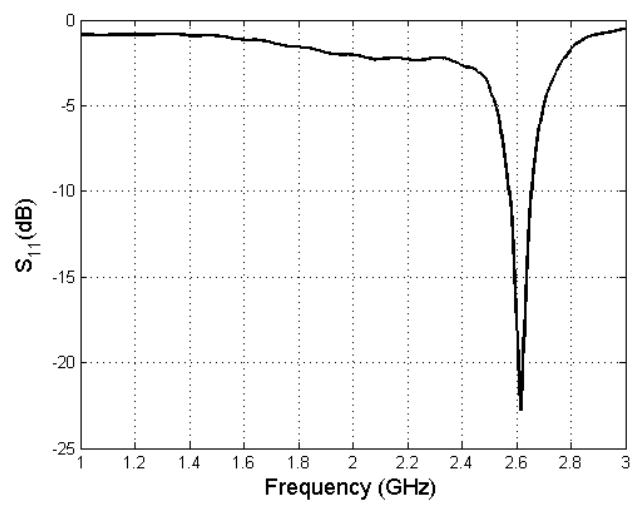

(b)

Fig. 4: Return loss of circular meshed patch antenna. (a) Antenna with mesh lines of $1.35 \mathrm{~mm}$,

(b) Antenna with mesh lines of $0.72 \mathrm{~mm}$.

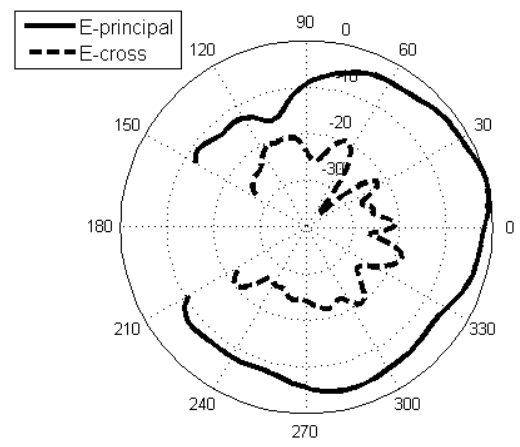

(a)

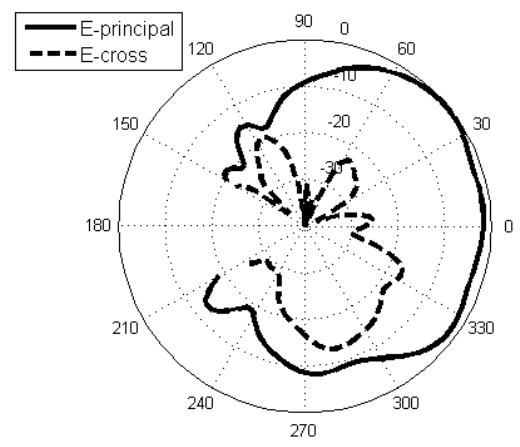

(b)

Fig. 5: Normalized radiation patterns of circular meshed patch antenna. (a) Antenna with mesh lines of $1.35 \mathrm{~mm}$, (b) Antenna with mesh lines of $0.72 \mathrm{~mm}$. 